\title{
TetR-family transcription factors in Gram- negative bacteria: conservation, variation and implications for efflux-mediated antimicrobial resistance
}

\author{
A. L. Colclough, J. Scadden and J. M. A. Blair *
}

\begin{abstract}
Background: TetR-family transcriptional regulators (TFTRs) are DNA binding factors that regulate gene expression in bacteria. Well-studied TFTRs, such as AcrR, which regulates efflux pump expression, are usually encoded alongside target operons. Recently, it has emerged that there are many TFTRs which act as global multi-target regulators. Our classical view of TFTRs as simple, single-target regulators therefore needs to be reconsidered. As some TFTRs regulate essential processes (e.g. metabolism) or processes which are important determinants of resistance and virulence (e.g. biofilm formation and efflux gene expression) and as TFTRs are present throughout pathogenic bacteria, they may be good drug discovery targets for tackling antimicrobial resistant infections. However, the prevalence and conservation of individual TFTR genes in Gram-negative species, has to our knowledge, not yet been studied.

Results: Here, a wide-scale search for TFTRs in available proteomes of clinically relevant pathogens Salmonella and Escherichia species was performed and these regulators further characterised. The majority of identified TFTRs are involved in efflux regulation in both Escherichia and Salmonella. The percentage variance in TFTR genes of these genera was found to be higher in those regulating genes involved in efflux, bleach survival or biofilm formation than those regulating more constrained processes. Some TFTRs were found to be present in all strains and species of these two genera, whereas others (i.e. TetR) are only present in some strains and some (i.e. RamR) are generaspecific. Two further pathogens on the WHO priority pathogen list ( $K$. pneumoniae and $P$. aeruginosa) were then searched for the presence of the TFTRs conserved in Escherichia and Salmonella.
\end{abstract}

Conclusions: Through bioinformatics and literature analyses, we present that TFTRs are a varied and heterogeneous family of proteins required for the regulation of numerous important processes, with consequences to antimicrobial resistance and virulence, and that the roles and responses of these proteins are frequently underestimated.

Keywords: TetR-family, Regulation, Antimicrobial resistance, Conservation

\footnotetext{
* Correspondence: J.m.a.blair@bham.ac.uk

Institute of Microbiology and Infection, Biosciences Building, University of

Birmingham, Edgbaston, Birmingham B15 2TT, UK
}

(c) The Author(s). 2019 Open Access This article is distributed under the terms of the Creative Commons Attribution 4.0 International License (http://creativecommons.org/licenses/by/4.0/), which permits unrestricted use, distribution, and reproduction in any medium, provided you give appropriate credit to the original author(s) and the source, provide a link to the Creative Commons license, and indicate if changes were made. The Creative Commons Public Domain Dedication waiver (http://creativecommons.org/publicdomain/zero/1.0/) applies to the data made available in this article, unless otherwise stated. 


\section{Background}

The TetR-family of transcriptional regulators (TFTRs) are a large family of one-component signal transduction proteins, with over 200,000 sequences available on public databases. TFTRs are implicated in the regulation of many processes, including efflux regulation, cell division and the stress response [1, 2]. Some of these processes are essential for cell growth and survival and therefore these TFTRs could be targets for inhibiting bacterial growth. Other processes, such as efflux, are important for antimicrobial resistance and the negative regulation of these efflux systems is commonly regulated by TFTRs.

TFTRs have a highly conserved helix-turn-helix (HTH) motif at the $\mathrm{N}$-terminus and a variable ligand-binding $\mathrm{C}$ terminal domain [3]. Many TFTRs act as repressors by binding palindromic sequences which overlap promoters, preventing the recruitment and binding of RNA polymerase and preventing transcription. Upon ligand binding, a conformational change occurs which releases the TFTR from target DNA, enabling transcription of target genes [2]. Some authors choose to classify TFTRs based on their location in relation to their target gene (Fig. 1) and it is believed that the majority of TFTRs regulate genes within 200 base pairs (bp) of the TFTR-encoding gene $[4,5]$. A TFTR classification system proposed by Ahn et al., describes three types of TFTR which bind targets which are either divergently encoded (Type I) encoded alongside (Type II) or neither I or II (Type III) [4]. Type I TFTRs are the most common (i.e. AcrR regulating $\operatorname{acr} A B$ ) than type II TFTRs (i.e. ComR regulating $\operatorname{com} A B$ ). Both Type I and II TFTRs are thought to act on local genes, whereas Type III TFTRs act globally and in any orientation (i.e. RutR).

There are numerous examples of TFTRs regulating local genes, such as AcrR regulating the adjacent $a c r A B$ efflux genes. However, some TFTRs are global regulators able to alter transcription of multiple targets throughout the genome, such as MtrR of Neisseria gonorrhoea [6]. In Mycobacteria the number of TFTRs has been shown to increase with genome size and while the number of TFTRs can vary between species, the majority of TFTRs in Mycobacteria are believed to regulate targets within $300 \mathrm{bp}$ of the tftr gene [5]. However, it is now known that some TFTRs act to regulate multiple targets and can therefore act locally and globally and meaning they would fit into multiple categories of the classification system in Fig. 1. For example, the TFTR EnvR regulates the divergently encoded local efflux operon acrEF, but also binds upstream and regulates expression of the efflux operon $\operatorname{acr} A B$, which is encoded separately on the genome. Some TFTRs with multiple targets may therefore not fit an individual classification of TFTR. Other TFTRs are activators [7] and some can act as both

Type I

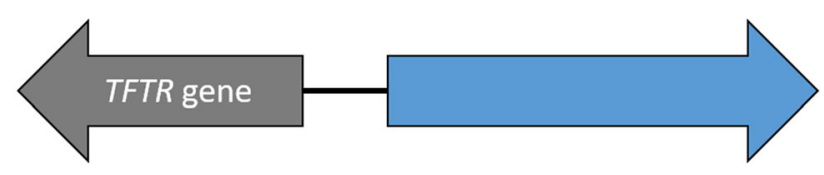

Type II

Or
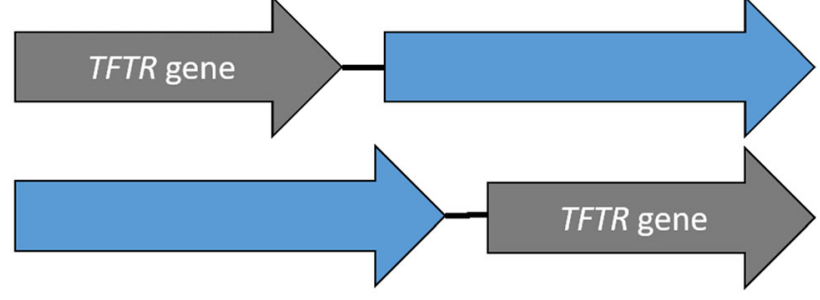

Type III

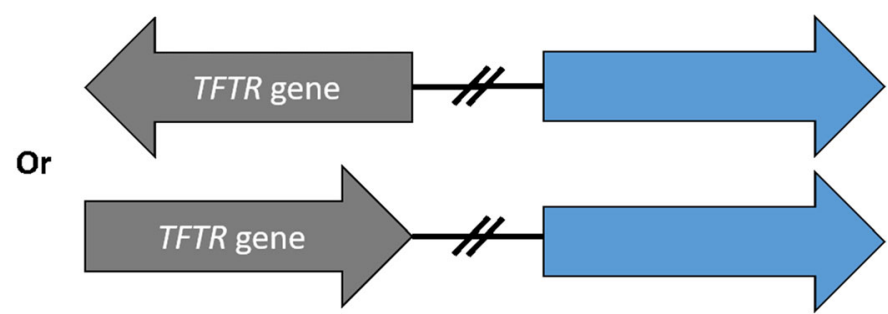

Fig. 1 TFTR regulation classification proposed by Ahn et al. Current classification system of TFTRs as proposed by Ahn et al. Type I classification involves the TFTR gene regulating a divergently expressed target gene (i.e. AcrR). Type II TFTRs regulate genes directly up/downstream in the same orientation (i.e. ComR). Type III TFTRs regulate genes either up/downstream of the TFTR gene in any orientation and any location on the genome 
activators and repressors [8]. TFTRs have been identified which can bind multiple targets $[9,10]$ and intergenic regions [11]. Thus, although some TFTRs are known to be local repressors, the current classification system is, in some cases, oversimplifying these proteins.

Efflux genes are frequently encoded in operons and are often negatively regulated by TFTRs. The extrusion of antimicrobials by efflux pumps such as $A$ crAB is a key mechanism of antimicrobial resistance [12, 13]. Specifically, mutations resulting in non-functional efflux regulators can cause increased expression of efflux genes, for example mutations in AcrR [14, 15] and EnvR [16], increase the efflux of antimicrobials by the AcrAB efflux system. These regulators may also have additional roles, for example there is evidence that AcrR can bind upstream of, and influence expression of $f l h C$ and $f l h D$, the master regulators of flagella expression [17].

While individual TFTRs have been studied in various Gram-negative bacteria and homologs of certain members of the TFTR family are known to be present in different species, it is not understood how conserved the TFTR family of proteins are across the Gram-negative bacteria, both in terms of which regulators and present/ absent or their level of sequence conservation.

Here, phylogenomic analyses of the conservation of the TFTRs across two genera, Escherichia and Salmonella were compared on three levels: genera, species and strain, to evaluate the conservation of TFTRs. From these analyses, we identify which TFTR genes are core (i.e. present in all) for Escherichia and Salmonella genera and of these core genes, which are also present in P. aeruginosa and K. pneumoniae. For this analysis, the TetR HTH was used to search for the presence of TFTR genes and then these genes were grouped based on function, through searching literature for experimental evidence of biological roles.

\section{Results}

Patterns of TFTR presence and absence across Escherichia and Salmonella genera

Maximum-likelihood trees constructed using the sequence of $a c r B$ were overlaid with data on the presence/absence of accessory TFTRs in the Escherichia and Salmonella genera using Phandango [18]. This data was combined with predicted function of these TFTRs, which was ascertained through searching known targets in the literature and compiled in Table 1, below:

The TFTRs identified here are included based on the presence of the TetR HTH motif. SImA contains this $\mathrm{HTH}$ and is therefore referred to by some authors as a TFTR. SlmA directly activates the transcription of the chb operon in $V$. cholerae [25], but is not believed to have any direct regulatory roles in E. coli [42]. In E. coli, $\operatorname{Sim} A$ acts as a nucleoid occlusion protein, interacting with target DNA and protein (FtsZ). Thus, although we include SlmA here, this is based on the presence of the $\mathrm{HTH}$ motif and not the assumption of direct regulatory roles in either Salmonella or Escherichia.

\section{TFTRs of E. coli and Escherichia species}

A median number of 14.5 TFTRs were identified in $E$. coli. Sequences of nemR, $\operatorname{sim} A, y b i H$, envR, acrR, uidR, rutR, fabR, betI and $y j d C$ were present in all strains of $E$. coli. A further six $(y t f A, t e t R, e e f R, y c f Q, y b j K$ and $y j g)$ were present in some, but not all strains of E. coli (Fig. 2). Strain SMS-3-5 contained the highest number of TFTRs $(n=16)$ and strain UTI89 the fewest $(n=12)$. A further two species within the Escherichia genera (three strains of E. albertii and two strains of E. fergusonii, Table 2, see methods) were searched for TFTR genes. These strains contained significantly fewer TFTRs than the E. coli strains (Student's t test $p<0.001$ ), with $E$. coli strains having an average TFTR number of 14 versus 10 for the E. albertii and E. fergusonii strains.

Six TFTRs (nemR, slmA, ybiH, envR, acrR and fabR) were present in all tested strains of the Escherichia genus. Of these regulators, the majority are involved in the removal of toxic compounds through either regulating efflux (AcrR, EnvR and YbiH) or, in the case of NemR, activating enzymatic pathways. The TFTRs uidR, bet $I$ and $y j d C$ were present in all $E$. coli strains, but were not present in all Escherichia strains searched. In contrast, these same three TFTRs were absent in all strains of $E$. fergusonii and $E$. albertii. In addition to these, all $E$. fergusonii strains also lacked eefR, $y c f Q$ and $y j g J$ and $E$. albertii strains lacked tetR. All strains of $E$. fergusonii and $E$. albertii have the $y t f A$ gene in all strains. In addition to these, E. albertii also have $y b j K$ and eefR and all strains of $E$. fergusonii have tetR. Both nodes containing E. fergusonii and E. albertii also contained fewer TFTRs per strain compared to E. coli.

\section{TFTRs of S. typhimurium and Salmonella species and serovars}

All strains of $S$. Typhimurium had 13 TFTRs and all but one strain, DT104, had the same TFTRs present (Fig. 2). The tet $R$ gene was present in DT104 but $y b j K$ was absent.

A further 9 strains of S. enterica of 7 different serotypes (Arizonae, Dublin, Enteritidis, Choleraesuis, Infantis, Newport, Paratyphi) and one strain of species S. bongori were searched for TFTRs. As with $S$. Typhimurium, the range of TFTRs in the Salmonella genus did not vary considerably $(n=12-14)$, with $S$. Choleraesuis strain SSCB67 having the most TFTRs $(n=14)$. Nine TFTRs acrR, envR, nemR, $\operatorname{sim} A, \operatorname{ramR}, \operatorname{rutR}, y c f Q, y j d C$ and $U 1$ were present in all strains of the Salmonella genus. As in Escherichia, the most frequent biological role of these core 
Table 1 Proposed biological roles of TFTRs of Salmonella and Escherichia. TFTRs present in all Gram-negative species tested are denoted as core**, while those not present in all species but present in all Escherichia and Salmonella are denoted as core*. The carriage of the remaining TFTRs found in Salmonella and Escherichia are listed (\%, italicised for Salmonella). This data is combined with biological role as documented in literature. Known targets and ligands are included and targets known to be activated, not repressed, by the TFTR are in bold. A biological role was assigned from the literature if experimental evidence was provided (e.g. binding assays to show TFTR binding to promoter)

\begin{tabular}{|c|c|c|c|c|c|}
\hline TFTR & Core/Accessory (\%) & Pathway & $\begin{array}{l}\text { Gene(s) or process regulated } \\
\text { (organism) }\end{array}$ & Ligands & References \\
\hline AcrR & Core $^{* *}$ & $\begin{array}{l}\text { Multidrug efflux (RND) } \\
\text { Multidrug efflux (ABC) } \\
\text { Multidrug efflux (MFS) } \\
\text { Motility }\end{array}$ & $a c r A B$ (Enterobacteriales) $\mathbf{f l} \mathbf{h D C}$ & $\begin{array}{l}\text { Rhodamine } 6 \mathrm{~g} \text { Proflavin Ethidium } \\
\text { bromide Ciprofloxacin }\end{array}$ & $\begin{array}{l}{[19]} \\
{[20]} \\
{[21]}\end{array}$ \\
\hline EnvR & Core $^{* *}$ & $\begin{array}{l}\text { Multidrug efflux (RND) } \\
\text { Multidrug efflux (RND) }\end{array}$ & $\begin{array}{l}\text { acrAB (Enterobacteriales) acrEF } \\
\text { (Enterobacteriales) }\end{array}$ & No data available & [9] \\
\hline NemR & Core $^{* *}$ & Bleach survival & $\operatorname{nem} A B$ & Choline & [22] \\
\hline $\operatorname{SImA}$ & Core* & $\begin{array}{l}\text { Cell division Chitin } \\
\text { catabolism }\end{array}$ & $\begin{array}{l}\text { FtsZ ring formation(Enterobacteriales) } \\
\text { chb operon (V. cholera) }\end{array}$ & $\begin{array}{l}\text { Target DNA sequences FtsZ } \\
\text { protein }\end{array}$ & $\begin{array}{l}{[23]} \\
{[24]} \\
{[25]}\end{array}$ \\
\hline YbiH & Core $^{*}$ & $\begin{array}{l}\text { Multidrug efflux (ABC) } \\
\text { Membrane permeability }\end{array}$ & ybhGFSR (E. coli) $\boldsymbol{r h l E}(E$. coli) & Chloramphenicol Cephalosporin & [26] \\
\hline Betl & Accessory (67\%) & $\begin{array}{l}\text { Glycine betaine } \\
\text { synthesis }\end{array}$ & $\begin{array}{l}\text { betT (Enterobacteriales) betIBA } \\
\text { (Enterobacteriales) }\end{array}$ & Choline & [27] \\
\hline EefR & Accessory (47\%) & Multidrug efflux (RND) & $\begin{array}{l}\text { eefABC (Enterobacter spp., K. } \\
\text { pneumoniae) }\end{array}$ & No data available & $\begin{array}{l}{[28]} \\
{[29]}\end{array}$ \\
\hline FabR & Core Accessory (93\%) & Fatty acid biosynthesis & fabAB (Enterobacteriales) & Unsaturated thioester & {$[30]$} \\
\hline RamR & Core & Efflux regulation & ramA (Enterobacteriales) & $\begin{array}{l}\text { Bile Berberine Ethidium bromide } \\
\text { Dequalinium Crystal violet } \\
\text { Rhodamine } 6 \mathrm{~g}\end{array}$ & $\begin{array}{l}{[31]} \\
{[32]} \\
{[33]}\end{array}$ \\
\hline RutR & Core Accessory (93\%) & $\begin{array}{l}\text { Pyrimidine utilisation } \\
\text { Purine degradation } \\
\text { Glutamine supply PH } \\
\text { homeostasis }\end{array}$ & $\begin{array}{l}\text { rutABCDEFG (E. coli) carAB (E. coli) } \\
\text { gadAXW (E. coli) gadIBC (E. coli) } \\
\text { gly-hyi-glXR-ybbVW-allB-ybbY-glxK } \\
\text { (E. coli) }\end{array}$ & Uracil Thymine & $\begin{array}{l}{[34]} \\
{[11]} \\
{[35]}\end{array}$ \\
\hline TetR & $\begin{array}{l}\text { Accessory (40\%) } \\
\text { Accessory (20\%) }\end{array}$ & Multidrug efflux (ABC) & tet $A B$ (Enterobacteriales) & Tetracycline & {$[36]$} \\
\hline UidR & Accessory (67\%) & $\begin{array}{l}\text { Catalysis of beta- } \\
\text { glucuronidase }\end{array}$ & uidA (E. coli) & No data available & {$[37]$} \\
\hline U1 & Core & No data available & No data available & No data available & \\
\hline $\begin{array}{l}\text { YbjK } \\
\text { RcdA }\end{array}$ & $\begin{array}{l}\text { Accessory (93\%) } \\
\text { Accessory (80\%) }\end{array}$ & $\begin{array}{l}\text { Biofilm formation } \\
\text { Stress response }\end{array}$ & $\begin{array}{l}\operatorname{csg} D \text { (E. coli) appY, sxy, ycgF, } \\
\operatorname{fimB}(\text { E. coli) }\end{array}$ & No data available & {$[38]$} \\
\hline $\begin{array}{l}\text { YcfQ/ } \\
\text { comR }\end{array}$ & Accessory (80\%) Core & Copper transport & comC (E. coli) & Copper & [39] \\
\hline YftA & Accessory (80\%) & No data available & No data available & No data available & \\
\hline YjdC & Accessory (67\%) Core & Copper tolerance & cadABC (E. coli) & No data available & {$[40]$} \\
\hline $\begin{array}{l}\text { YjgJ/ } \\
\text { bdcR }\end{array}$ & $\begin{array}{l}\text { Accessory (60\%) } \\
\text { Accessory (93\%) }\end{array}$ & Biofilm dispersal & $b d c A(E$. coli) & No data available & [41] \\
\hline
\end{tabular}

TFTRs is efflux regulation, with 3 core TFTRs of Salmonella (AcrR, EnvR and RamR) being involved in the regulation of multidrug efflux systems. Two TFTR genes were identified ( $\mathrm{ramR}$ and U1) which were not present in any Escherichia spp. strain in this study. All nodes of the Salmonella tree contained the same TFTRs apart from $S$. arizonae which lacked yjgJ. This is unsurprising as most Salmonella strains included here are serovars within the $S$. enterica species and do not show large variation in either the number or type of TFTRs.
TFTR number increases with genome size $(\mathrm{Mb})$

The number of bacterial regulators is known to increase with genome size [1] and TFTR number is known to be positively correlated with genome size in Mycobacteria [5]. Here, we show that TFTR number is significantly positively correlated with genome size for a range of bacterial species $\left(\mathrm{R}^{2}=0.85, p<0.01\right)$ (Fig. 3). The median genome sizes and TFTR numbers in this study were also comparable to the large number of genomes deposited on the NCBI database (Fig. 3b), validating the 

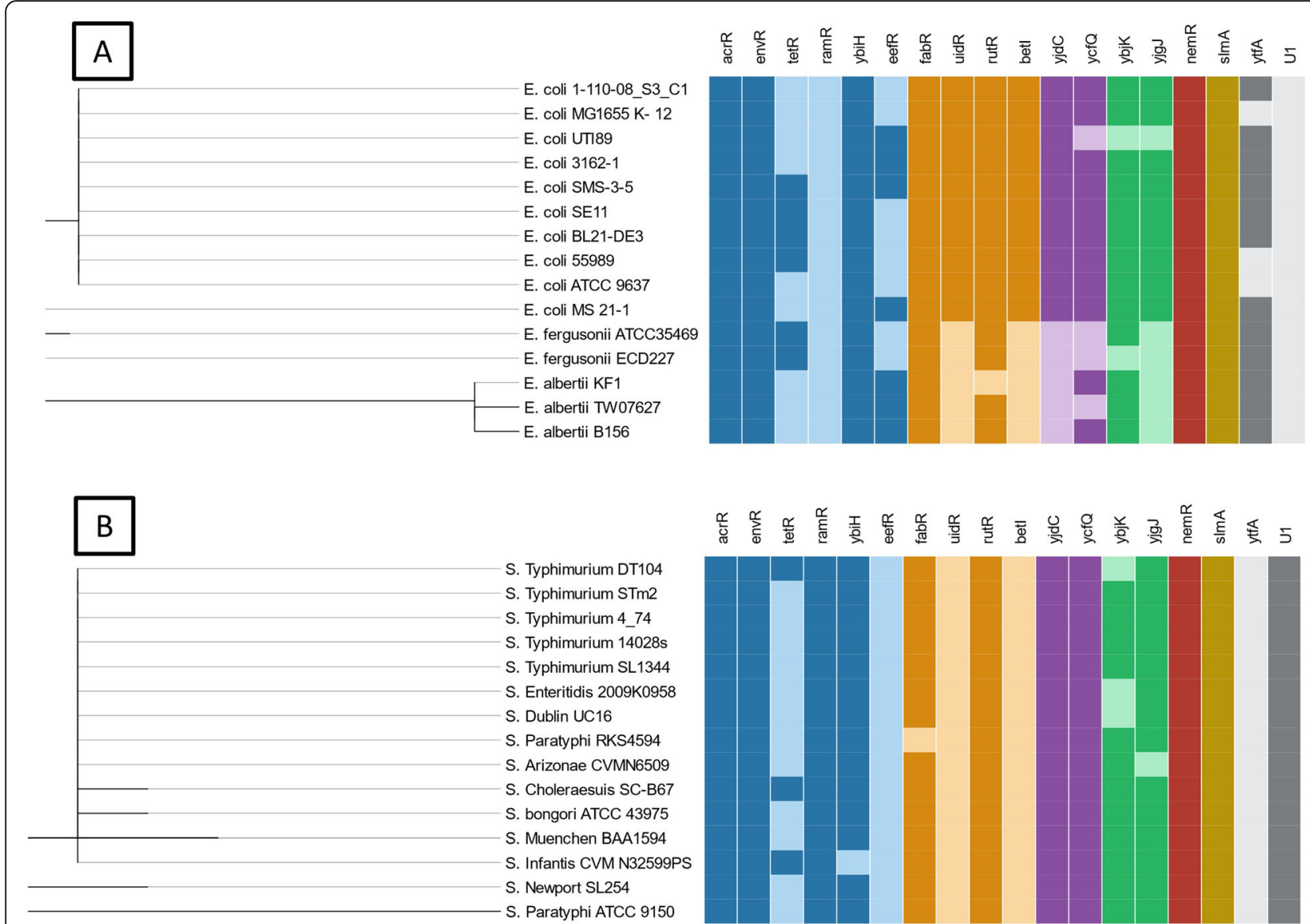

Fig. 2 Patterns of TFTR presence/absence across Escherichia and Salmonella strains. TFTR presence/absence across strains of Escherichia (a) and Salmonella (b). Colours of squares indicate proposed function of TFTR, with darker colours indicating presence of the gene in the given strain and lighter colours indicating the gene is absent

methodology used here. $P$. aeruginosa has both the largest median genome size and predicted TFTR number (median $=39$, range 36-45). All $S$. Typhimurium strains had 13 TFTRs whereas the Salmonella genera had a small range of 12-14 TFTRs. E. coli strains had a slightly larger range of 12-16 TFTRs than Salmonella and the Escherichia genus as a whole had a range of 9-16 TFTRs. There was a significant difference in the number of TFTRs found in E. albertii and E. fergusonii versus $E$. coli and Pseudomonas spp. versus $P$. aeruginosa, with the $E$. coli and $P$. aeruginosa strains having a higher number of TFTRs. It is not known whether the number of targets of TFTRs also increases in larger genomes. As many TFTRs have multiple targets this is difficult to ascertain, and it is also possible that targets for individual TFTRs vary between bacterial species.

\section{Biological roles of TFTRs of Escherichia and Salmonella} There were five TFTR genes found in all Salmonella and Escherichia searched here: [1] Bleach response regulator
nemR, Efflux regulators [2] acrR, [3] envR and [4] ybiH and nucleoid occlusion factor [5] $\operatorname{sim} A$. In order to classify the TFTRs by role, existing literature was searched for evidence of the regulatory targets and ligands of all TFTRs identified in Escherichia and Salmonella. Efflux regulation was the most frequent TFTR function $(n=6)$ and the majority of TFTRs which are core in both Salmonella and Escherichia are efflux regulators. Escherichia spp. had two extra TFTRs which regulate metabolism, but there were no other differences in the distribution of TFTR role between these genera (Fig. 4).

Data on the function of TFTRs was then combined with data on the presence/absence of these genes throughout the Escherichia and Salmonella genera (Table 1). In addition to the five genes conserved in all Gram-negatives tested here $(a c r R, \operatorname{env} R, n e m R, \operatorname{sim} A$ and $y b i H)$, two were core to Escherichia (fabR and rutR) and a further four $(\mathrm{ramR}, U 1, y c f Q$ and $y j d C)$ were core for Salmonella. Nine TFTRs are, based on current available literature, singletarget regulators. A further seven TFTRs have been shown 
Table 2 Salmonella and Escherichia strains in this study. The nomenclature (genus, species, serovar and strain), accession and number of TFTR sequences are listed for all strains of Salmonella and Escherichia in this study

\begin{tabular}{|c|c|c|c|c|}
\hline Genus & Species/ species and serovar & Strain & NCBI Tax ID & Number of IPR001647 hits \\
\hline Salmonella & enterica serovar Typhimurium & DT104 & 85,569 & 13 \\
\hline Salmonella & enterica serovar Typhimurium & STm2 & $1,218,144$ & 13 \\
\hline Salmonella & enterica serovar Typhimurium & 4_74 & 909,946 & 13 \\
\hline Salmonella & enterica serovar Typhimurium & $14,028 s$ & 588,858 & 13 \\
\hline Salmonella & enterica serovar Typhimurium & SL1344 & 216,597 & 13 \\
\hline Salmonella & enterica serovar Enteritidis & 2009 K0958 & $1,192,586$ & 12 \\
\hline Salmonella & enterica serovar Dublin & UC16 & $1,192,688$ & 12 \\
\hline Salmonella & enterica serovar Paratyphi & RKS4594 & 476,213 & 12 \\
\hline Salmonella & enterica serovar Arizonae & CVMN6509 & $1,395,108$ & 12 \\
\hline Salmonella & enterica serovar Choleraesuis & SC-B67 & 321,314 & 14 \\
\hline Salmonella & bongori & ATCC 43975 & 54,736 & 13 \\
\hline Salmonella & enterica serovar Muenchen & BAA1594 & $1,079,477$ & 13 \\
\hline Salmonella & enterica serovar Infantis & CVM N32599PS & $1,439,843$ & 13 \\
\hline Salmonella & enterica serovar Newport & SL254 & 423,368 & 13 \\
\hline Salmonella & enterica serovar Paratyphi & ATCC 9150 & 295,319 & 13 \\
\hline Escherichia & coli & 55,989 & 585,055 & 14 \\
\hline Escherichia & coli & ATCC 9637 & 566,546 & 13 \\
\hline Escherichia & coli & BL21-DE3 & 469,008 & 15 \\
\hline Escherichia & coli & MS 21-1 & 749,527 & 15 \\
\hline Escherichia & coli & SE11 & 409,438 & 15 \\
\hline Escherichia & coli & SMS-3-5 & 439,855 & 16 \\
\hline Escherichia & coli & $3162-1$ & $1,281,200$ & 15 \\
\hline Escherichia & coli & UT189 & 364,106 & 12 \\
\hline Escherichia & coli & 1-110-08_S3_C1 & $1,444,132$ & 14 \\
\hline Escherichia & coli & MG1655 K-12 & 511,145 & 13 \\
\hline Escherichia & albertii & TW07627 & 502,347 & 10 \\
\hline Escherichia & albertii & B156 & 550,693 & 11 \\
\hline Escherichia & albertii & KF1 & $1,440,052$ & 10 \\
\hline Escherichia & fergusonii & ATCC35469 & 585,054 & 10 \\
\hline Escherichia & fergusonii & ECD227 & 981,367 & 9 \\
\hline
\end{tabular}

to either bind upstream of, or affect the transcription of, multiple genes. RutR and $\mathrm{YbjK}$ are known activators of at least one of their target genes $[11,35]$. Nucleoid occlusion factor SImA has no known transcriptional regulatory activity in $E$. coli but is a known activator in $V$. chloerae [25].

Certain TFTRs are genera-specific, e.g. the eefR gene was not present in any Salmonella strains and ramR is absent in Escherichia strains. TFTRs conserved throughout a genera are denoted as 'core' and all other TFTRs are therefore 'accessory' for this same genera. Therefore Salmonella and Escherichia have their own set of core and accessory TFTRs. The percentage carriage of each accessory TFTR was calculated for strains of both genera. Strains lacking the eefR gene were also found to lack eefA and eefB, components of the EefABC efflux system in Enterobacter (Additional file 1).

We were unable to collect information on two regulators (YftA and U1) and the sequences of the unidentifiable TFTR are in Additional file 2.

\section{Sequence variation is related to predicted biological function}

The biological roles of many TFTRs in this study are known in E. coli, but it is not known if the targets, ligands or functions of TFTRs are genera, species or even strain-specific.

TFTRs which regulate efflux, bleach survival and biofilm formation and dispersal have significantly higher percentage variance (Student's t test $p=0.01$ ) than those 

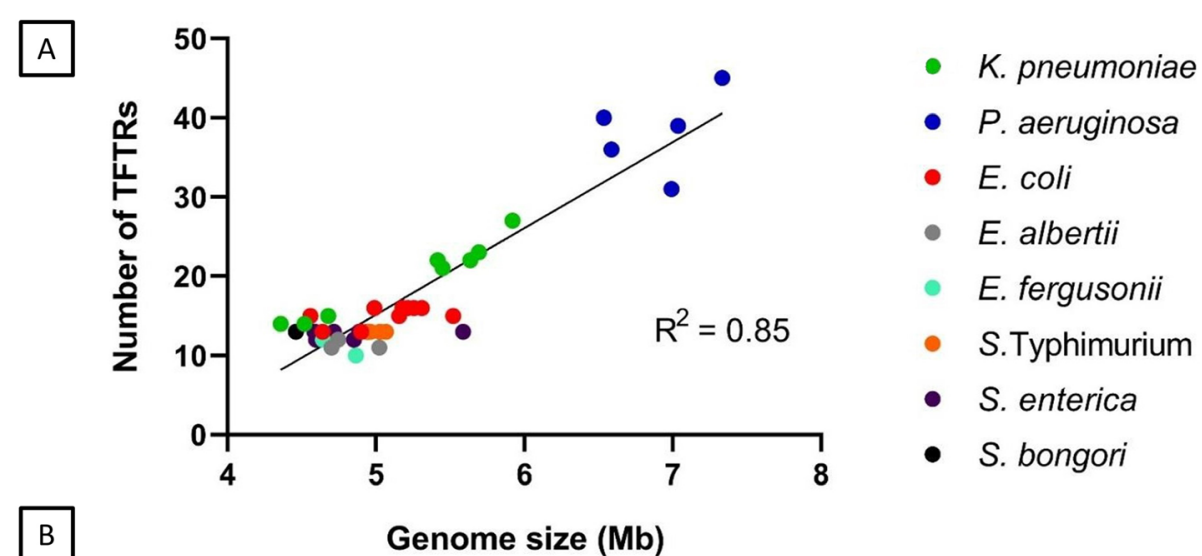

Genome size (Mb)

\begin{tabular}{|l|l|l|l|l|l|l|}
\hline Organism & $\begin{array}{l}\text { NCBI median } \\
\text { genome size } \\
(\mathrm{Mb})\end{array}$ & $\begin{array}{l}\text { NCBI No. } \\
\text { Genome } \\
\text { assemblies }\end{array}$ & $\begin{array}{l}\text { Median } \\
\text { genome size } \\
\text { (This study, } \\
\text { Mb) }\end{array}$ & $\begin{array}{l}\text { No. Genomes } \\
\text { (This study) }\end{array}$ & Per Mb & $\begin{array}{l}\text { Median } \\
\text { predicted TFTR } \\
\text { no. }\end{array}$ \\
\hline P. aeruginosa & 6.6 & 3,018 & 6.7 & 10 & 5.8 & 39 \\
\hline K. pneumoniae & 5.6 & 6,101 & 5.4 & 10 & 3.8 & 21 \\
\hline Escherichia spp. & - & - & 5.0 & 5 & 2.2 & 11 \\
\hline E. coli & 5.1 & 13,288 & 5.2 & 10 & 2.9 & 15.5 \\
\hline Salmonella spp. & 4.8 & 9,436 & 4.9 & 10 & 2.6 & 13 \\
\hline S. Typhimurium & - & - & 5.0 & 5 & 2.6 & 13 \\
\hline A. baumanii & 4.0 & 3,254 & 4.1 & 10 & - & - \\
\hline
\end{tabular}

Fig. 3 Genome size is positively correlated with the number of TFTRs. a TFTR number varied between strains, species and genera of bacteria but was significantly positively correlated with genome size (Mb). The largest range of TFTR number was seen in Pseudomonas spp. and the smallest in S. Typhimurium. $\mathbf{b}$ Table describes median genome sizes and $n=$ TFTRs in this study versus NCBI database. The median genome sizes were compared to genomes in this study to check that the genomes selected had a median genome size which is representative of the wider population of isolates. The number of predicted TFTRs was calculated by searching Interpro for IPR001647-containing sequences as previously described. A full list of strains used to produce this figure are available in Additional file 1 and data used to create this figure can be found in Additional file 3

involved in regulating cell division, metabolism or copper transport. There was no significant difference in level of TFTR variation between Escherichia and Salmonella. The lowest variance was seen in nucleoid occlusion factor SlmA.

\section{Sequence variation is gene and organism- dependant}

As the sequence variation of TFTRs was shown to vary due to function (Fig. 5), the percentage variation in the TFTR target genes was also investigated and compared to variability of the regulator, in order to ascertain if this could be a function or regulatorspecific effect. The percentage variation in TFTRs is shown below (Fig. 6).

There was no clear pattern in how level of variation in the regulator sequence relates to variation in target gene sequence. Sequences of $a c r R$ were more varied than the operon it regulates, $\operatorname{acr} A B$, whereas $f a b R$ was less variable than $f a b A B$. The amount of variation seen in a regulator and its target(s) also varied between genera. For example, there was higher variation in the acrEF-envR sequences in Escherichia. However, for most other regulator/target pairs, such as fabR- $f a b A B$, there were no differences between the genera. Some gene homologs may therefore be under similar levels of selective pressure resulting in comparable levels of variance in different genera.

\section{Discussion}

The number of genes encoding transcription factors varies between bacterial species and this variation depends on both genome size and bacterial lifestyle, with small-genome, niche-restricted species having fewer transcriptional regulators [43, 44]. Conversely, bacteria with large genomes and varied lifestyles such as Pseudomonas species contain the largest number of regulatory genes of bacterial genomes studied to date [45]. Data here supports the observation by others that TFTR number positively correlates with genome size [5] and shows that this trend exists throughout Escherichia and Salmonella in addition to other Gram-negative species.

The inclusion of pathogenic, environmental and laboratory strains, makes the results reported here more representative of the genera as a whole. Strains and species of Salmonella and Escherichia show variation in the number of TFTRs present, thus even the most recent of 


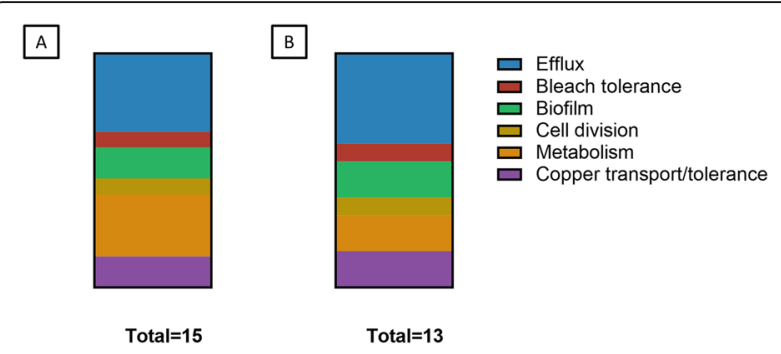

Fig. 4 Biological roles of TFTRs in Escherichia and Salmonella. Proportion of TFTRs predicted to regulate various processes in (a) Escherichia spp. and (b) Salmonella spp. Based on the function assigned from literature search (Table 1). Escherichia spp. have two additional TFTRs involved in regulating metabolism. No other differences between TFTR function in Escherichia and Salmonella were observed

evolutionary events are selecting for or against the conservation of certain TFTR genes.

Salmonella species tested here were (aside from one strain) serovars of species S. enterica and therefore it is expected that these strains did not show significantly different TFTR numbers. However, the E. coli strains had significantly more TFTRs than the other species in the genus, $E$. fergusonii and $E$. albertii. All three of these Escherichia species have broad host ranges as they are able to colonise and cause infections in both humans and animals [46, 47]. Both E. fergusonii and E. albertii are emerging enteropathogens [48] [49]. It is possible that the differences in regulatory genes reflect the different lifestyles and virulence of these species.

TFTRs are frequently thought of as simple, singletarget negative regulators, however, some have been shown to have multiple targets (e.g. EnvR) [9]. Some TFTRs can be both activators and/or repressors (e.g. MtrR and MerR) or can repress or activate multiple targets (e.g. $g \ln E$ [6] [50]). Recent work by Shimada et al, demonstrates that, for multiple classes of transcription factors, single-target function may be the exception, not the rule [51].

Of the three TFTRs found to be core across the Gram-negative species studied (AcrR, EnvR and NemR), two are regulators of efflux (AcrR and EnvR) and the other promotes bleach tolerance (NemR). This was surprising as it was expected that TFTRs which regulated processes with implications for virulence would likely vary more throughout Gram-negative bacteria. It has been reported that up to $25 \%$ of known TFTRs act as regulators of efflux systems [4]. Consistent with this, $33 \%$ of TFTRs were predicted to regulate efflux systems in this study. However, when only considering TFTRs found in all strains the majority were efflux regulators. Thus, the most widespread TFTRs in Gram-negative bacteria are those involved in efflux regulation. Efflux is a key mechanism of antimicrobial resistance and the ability to overexpress efflux systems can confer multidrug resistance, therefore understanding the TFTR regulators of these efflux pumps is essential to better understanding efflux-mediated resistance.

It is possible that the prevalence of TFTRs conserved in this dataset was skewed due to the selection of strains from the WHO priority pathogens list of multidrug resistance species, or perhaps the processes regulated by core TFTRs (i.e. efflux) are more widespread than previously thought. However, the strains used in this study originate from multiple sources (including patient samples, environment and laboratory strains). The number

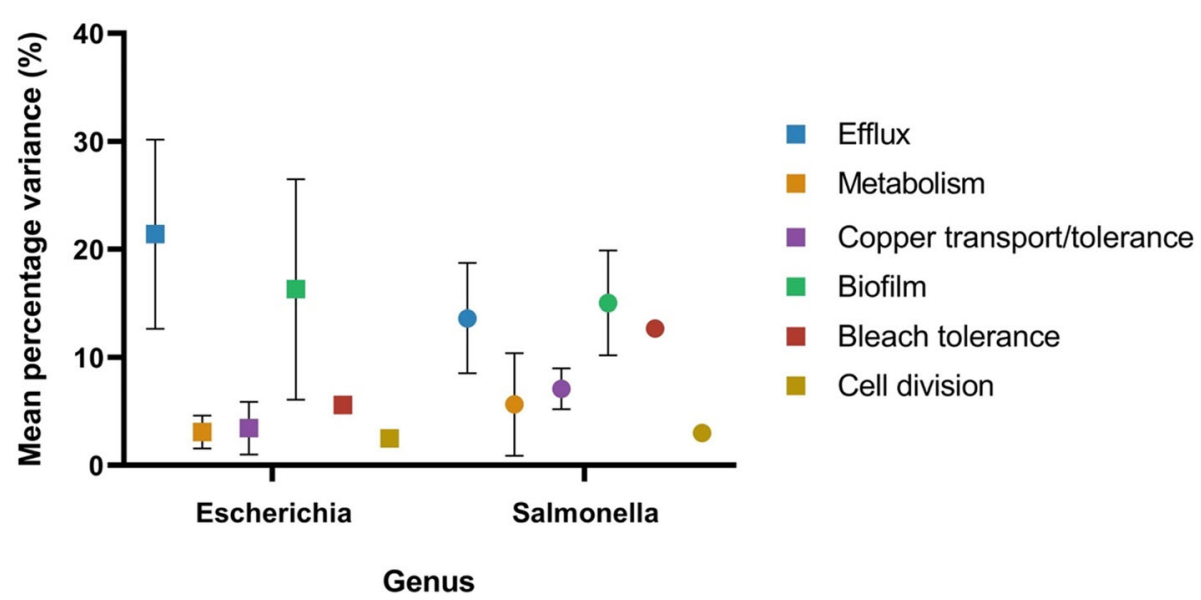

Fig. 5 Mean percentage variation in TFTRs grouped by biological function. Percentage sequence variation of TFTRs grouped by function with standard error of the mean. TFTRs regulating efflux regulation, bleach survival or biofilm formation/dispersal have significantly higher percentage variance (Student's t test $p=0.01$ ) than those involved in cell division, metabolism or copper transport/tolerance. This was not a generadependant effect, with no significant difference between percentage variance of TFTRs between Escherichia and Salmonella genera 


\section{Escherichia spp.}

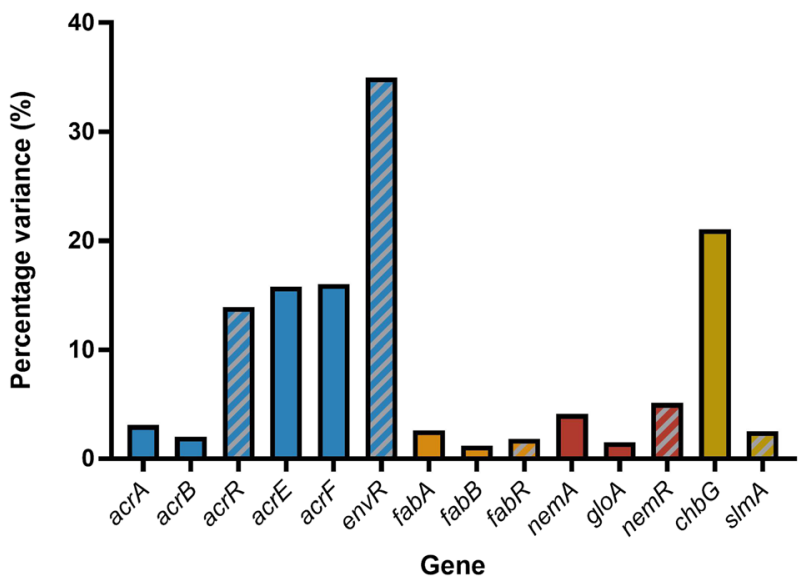

Salmonella spp.

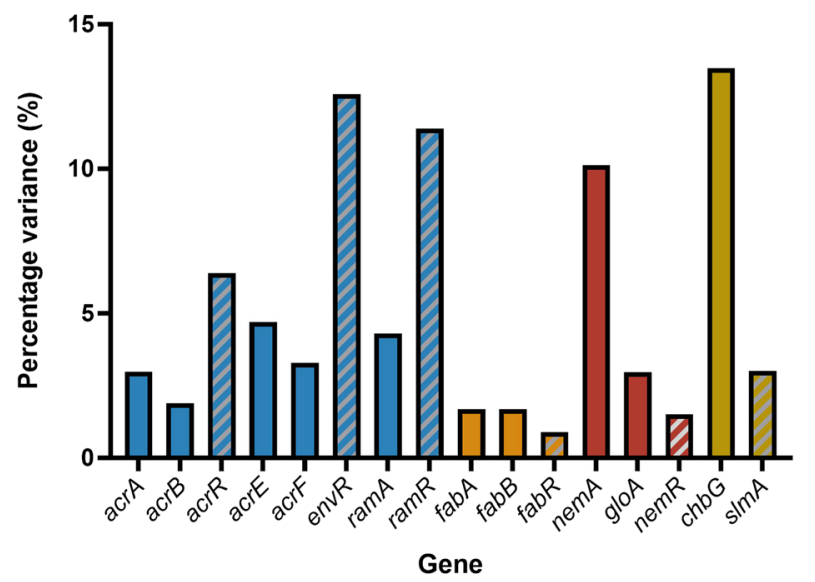

Fig. 6 Percentage sequence variation in TFTRs and their targets. Percentage variation in amino acid sequence in TFTRs and their target genes in Salmonella and Escherichia generated from the sum of polymorphisms after alignment of sequences of the listed genes for each genera

of targets and functions of a TFTR may also either influence the carriage of a particular TFTR gene.

Homologous transcriptional regulators may evolve differentially in different species allowing the acquisition or loss of targets and therefore the further specialisation of the regulator [52]. This means that it is not only the number, but the function, targets and sequence similarity of TFTRs and other regulators which is likely to vary between bacterial species. For example, in one species there may be selective pressure to gain function (i.e. to allow a TFTR to gain an additional target) and it may be expected that some TFTRs have evolved to gain/lose function in accordance with the specificity of the target gene(s) they regulate. The notion that transcriptional regulators can gain targets is not new, for example the CRP regulon of $E$. coli $\mathrm{K}-12$ can be observed to evolve under laboratory conditions in just over 20,000 generations [53].

The plasticity of regulatory pathways is thought to exist partly due to duplication events, through which regulatory genes are duplicated and undergo subsequent specialisation in function [54]. This could also explain some redundancies in regulatory targets and binding sites of TFTRs (for example, AcrR and EnvR both bind to the same site upstream of acrAB). The cross-talk of these efflux systems is not well understood but understanding the conservation of these genes gives insight as to their importance in bacterial species. Moreover, understanding the multiple regulators involved in regulating RND efflux could provide opportunity for drug discovery targets to be identified.

Classifying roles of TFTRs using published literature had unpredicted consequences for this study, including the identification of numerous pseudogenes (Additional file 1 ) and the identification of the EefABC efflux system in some Escherichia species. The TFTR EefR regulates the EefABC RND efflux system in Enterobacter species, which is also under regulation by H-NS $[28,29]$. This efflux system has not, to our knowledge, been reported in Escherichia species. The gene coding for the regulator of the efflux system, eefR, was found in four $E$. coli strains and all three E. albertii strains, potentially also indicating that the EefABC efflux system is present in these strains of E. coli and may therefore be present in other bacterial species.

Higher percentage variation was seen in TFTRs which regulate processes which contribute towards antimicrobial resistance or virulence (i.e biofilm dispersal and efflux) compared to other TFTRs. This may be because variation here can confer favourable phenotypes, which promotes dissemintation and eventually, fixation, of these genotypes. For example, when challenged with antimicrobials, mutations which cause loss-of-function of the TFTR regulator are selected. Polymorphisms in efflux regulators AcrR [14, 15], EnvR [16], RamR [55] and TetR [56], have been reported previously and in this study premature stop codons were observed in the sequences of envR, acrR, acrE and acrF (Additional file 1).

Patterns in sequence variation were not replicated in the target genes of the TFTRs, i.e. the efflux genes tested did not have significantly higher percentage variation than other target genes. This indicates that it is the regulators themselves which are under either positive or negative selective pressure based on the target(s) they regulate and not the particular operon (in the case of locally-acting TFTRs), location with regards to the origin of replication or location within pathogenicity islands. Sequences of $a c r R$ and $a c r A B$ showed a similar pattern in both Escherichia and Salmonella strains, with higher variation in the sequence of the regulator. Similarly, 
variation in $f a b R$ and $f a b A B$ remained low in both genera. Variation was higher in general in Escherichia species, although strains within this group were more genetically distant than those tested in the Salmonella genera. Some patterns of TFTR and target variation did vary between the genera, notably there was much higher variation in sequences of envR and acrEF in Escherichia. The AcrEF efflux pump shares many substrates with AcrAB and the acrEF operon is thought to be H-NS silenced under most conditions [9]. The operon may not be required in many situations, meaning that the whole region encounters spurious polymorphisms and genetic drift.

\section{Conclusions}

The conservation and heterogeneity of TFTRs discussed here highlights the varied and sometimes, underestimated, roles of TFTRs. TFTRs which regulate processes promoting pathogenicity, virulence or multidrug resistance are likely to be more ubiquitous, but contain more sequence variation, throughout Gram-negative bacteria. Our current understanding of TFTRs is largely based on those we have characterised well and for which we have crystal structures, but often leads to the misunderstanding that all TFTRs act in these more simplistic ways.

We propose that the current classification system of TFTRs underestimates the roles of TFTRs and that these proteins often regulate many targets, sometimes using multiple mechanisms.

This is, to our knowledge, the first wide-scale study on TFTRs across Gram-negative pathogens. With rising levels of antimicrobial resistance and limited novel treatment options, we should seek to better understand regulators such as TFTRs which are frequently implicated in multidrug resistant phenotypes.

\section{Methods}

Identification of TFTR genes in Escherichia and Salmonella

TFTRs contain a highly conserved helix-turn-helix (HTH) domain at the $\mathrm{N}$-terminus which is denoted as IPR001647 on EMBL-EBI Interpro [57]. Available deposited proteomes of Salmonella enterica serovar Typhimurium and Escherichia coli (5 strains of S. Typhimurium and 10 strains of E. coli) were searched for this conserved domain and these protein sequences downloaded. This approach rapidly provides a proxy for how many TFTRs are present due to the high conservation of the HTH domain. Where possible, sequences were annotated with protein name. All proteins had their annotation manually curated using pBLAST [58], producing a database of TFTR protein sequences with confirmed annotations. Orthologues were aligned using Clustal OMEGA [59, 60] to produce neighbour-joining trees of all TFTRs of $S$. Typhimurium and E. coli. For example, the sequence of $b m 3 R 1$ shared
$100 \%$ identity with $\operatorname{ramR}$ and clustered with other $\operatorname{ram} R$ sequences, but without this alignment these sequences may have been incorrectly assigned an individual identity. This approach also helped to ensure that proteins with multiple names in use (i.e. NemR/ YdhM and ComR/YcfQ) were identified as one group and not duplicated.

In order to investigate the variation in TFTR number, type and sequence identity, more proteomes of the wider genera (Salmonella and Escherichia) were searched in the same way as described above. Table 2 lists all Salmonella and Escherichia strains included in this study. Any unannotated proteins were searched on pBLAST and all putative TFTRs were aligned with the confirmed ID TFTRs of either S. Typhimurium SL1344 or E. coli K- 12. TFTRs present in all strains of Salmonella or Escherichia were denoted as 'core' for the given genera. TFTR differentially present in our analysed dataset were denoted as 'accessory'. Table 1 reviews the known and suspected biological roles of all identified TFTRs and lists whether each TFTR identified was core or accessory for Salmonella and Escherichia.

\section{TFTRs in other Gram-negative species}

The WHO priority pathogen list comprises the pathogens which most urgently require new antibiotics due to the emergence of multidrug resistant isolates. Salmonella and Escherichia species are on this list alongside other clinically relevant species such as $P$. aeruginosa and $K$. pneumoniae. The total number of IPR001647 containing-sequences were recorded alongside data on median genome length provided on NCBI. Proteomes, and not genomes, were selected for analyses in this study to enable searching for the specific HTH of TFTRs to prevent false positives. The Gram-negative strains used are listed in Additional file 1.

\section{Sequence variation of TFTRs and their regulated genes}

In order to investigate the variability of TFTRs, all sequences of TFTRs in Escherichia and Salmonella ( $n=$ 384) were aligned using Clustal Omega and percentage sequence variation was calculated as the sum of the variable amino acid positions across all sequences of a particular gene in a genera divided by average length of the TFTR gene (Fig. 5). Sequence length was therefore accounted for when considering percentage amino acid variation and TFTRs were grouped based on functions assigned in Table 1.

Known and suspected targets of all the TFTRs identified in Salmonella and Escherichia spp. were curated by searching available published literature (Table 1). The amino acid sequences of each TFTR were compiled and aligned using Clustal Omega and the number of variable amino acid positions counted. This total was then 
divided by the mean sequence length for a given TFTR to account for variations in TFTR gene length and multiplied by 100 to give the percentage sequence variance. Here, a variable amino acid position was defined as a position with no consensus amino acid, denoted as either blank, " or ** on Clustal Omega, depending on the possible amino acid substitutions. This process was repeated for the known targets genes of the core TFTRs, excluding targets without conclusive binding studies (i.e. Electrophoretic mobility shift assay) or other proof of binding or regulation (i.e. transcriptomics, ChIP/RNAseq).

\section{Phylogenetic analyses}

A multiple sequence alignment of the amino acid sequence of AcrB was constructed using MUSCLE [61] for all strains of Salmonella and Escherichia in this study. The sequence of AcrB varies between strains of Escherichia and Salmonella and was therefore an ideal candidate for clustering our strains to our desired level of depth. This alignment was then used to construct maximum-likelihood trees with a 100 bootstrap cut-off using MEGA7 [62]. The primary aim of these trees was to separate a small number of very closely-related strains in order to map whether specific TFTRs are present/absent in species of each genera, not precisely map the evolutionary distance between these strains in depth. Phandango was used to combine metadata with the phylogenetic analysis from MEGA7 [18].

\section{Additional files}

Additional file 1: Supplementary material detailing (A) identified pseudogenes, (B) Strains of $K$. pneumoniae and $P$. aeruginosa used in this study and (C) Evidence that strains lacking eefR also lack eefABC. (PDF 673 kb)

Additional file 2: Supplementary material listing all the unidentified TFTRs from this study. (XLSX $11 \mathrm{~kb}$ )

Additional file 3: Supplementary data containing raw data used to produce Figs. 3 and 6. (XLSX 78 kb)

\section{Abbreviations}

Bp: Base pairs; HTH: Helix-turn-helix; RND: Resistance nodulation division family of efflux pumps; TFTRs: TetR- family transcriptional regulators

\section{Acknowledgements}

Thank you to both Professor Alan McNally and Professor David Grainger who read, commented and advised on the manuscript prior to submission.

\section{Authors' contributions}

JS and ALC collected and analysed the data. ALC interpreted data. JMAB supervised, helped with data interpretations, idea generation. ALC and JMAB wrote the manuscript. All authors read and approved the final manuscript.

\section{Funding}

This work was funded by BBSRC grant BB/M02623X/1 (David Phillips Fellowship to JMAB). ALC is supported by a University of Birmingham PhD Studentship. Funding bodies did not contribute towards the [1] the design of the study, [2] data collection, analysis or interpretation or [3] writing of the manuscript.

\section{Availability of data and materials}

All data generated or analysed during this study are included in this published article and its supplementary information files.

Ethics approval and consent to participate

Not applicable.

\section{Consent for publication}

Not applicable.

\section{Competing interests}

The authors declare that they have no competing interests.

Received: 27 March 2019 Accepted: 30 August 2019

Published online: 12 October 2019

References

1. Cuthbertson L, Nodwell JR. The TetR family of regulators. Microbiol Mol Biol Rev. 2013;77(3):440-75

2. Ramos JL, Martinez-Bueno M, Molina-Henares AJ, Teran W, Watanabe K, Zhang $X$, et al. The TetR family of transcriptional repressors. Microbiol Mol Biol Rev. 2005;69(2):326-56.

3. Orth P, Schnappinger D, Hillen W, Saenger W, Hinrichs W. Structural basis of gene regulation by the tetracycline inducible Tet repressor-operator system. Nat Struct Biol. 2000;7(3):215-9.

4. Ahn SK, Cuthbertson L, Nodwell JR. Genome context as a predictive tool for identifying regulatory targets of the TetR family transcriptional regulators. PLoS One. 2012;7(11):e50562.

5. Balhana RJ, Singla A, Sikder MH, Withers M, Kendall SL. Global analyses of TetR family transcriptional regulators in mycobacteria indicates conservation across species and diversity in regulated functions. BMC Genomics. 2015;16:479.

6. Johnson PJT, Shafer WM. The transcriptional repressor, MtrR, of the mtrCDE efflux pump operon of Neisseria gonorrhoeae can also serve as an activator of "off target" gene (glnE) expression. Antibiotics. 2015;4(2):188-97.

7. Hu B, Lidstrom M. CcrR, a TetR family transcriptional regulator, activates the transcription of a gene of the Ethylmalonyl coenzyme a pathway in Methylobacterium extorquens AM1. J Bacteriol. 2012;194(11):2802-8.

8. Chattoraj P, Mohapatra SS, Rao JL, Biswas I. Regulation of transcription by SMU.1349, a TetR family regulator, in Streptococcus mutans. J Bacteriol. 2011;193(23):6605-13.

9. Hirakawa H, Takumi-Kobyashi A, Theisen U, Nishino K, Yamaguchi A. AcrS/ EnvR represses expression of the acrAB multidrug efflux genes in Escherichia coli. J Bacteriol. 2008;190(18):6276-9.

10. Liu H, Yang M, He ZG. Novel TetR family transcriptional factor regulates expression of multiple transport-related genes and affects rifampicin resistance in Mycobacterium smegmatis. Sci Rep. 2016;6:27489.

11. Shimada T, Ishihama A, Busby SJ, Grainger DC. The Escherichia coli RutR transcription factor binds at targets within genes as well as intergenic regions. Nucleic Acids Res. 2008;36(12):3950-5.

12. Okusu $H, M a D$, Nikaido $H$. AcrAB efflux pump plays a major role in the antibiotic resistance phenotype of Escherichia coli multiple-antibioticresistance (mar) mutants. J Bacteriol. 1996;178(1):306-8.

13. Nikaido $H$, Pages JM. Broad-specificity efflux pumps and their role in multidrug resistance of gram-negative bacteria. FEMS Microbiol Rev. 2012;36(2):340-63.

14. Adler M, Anjum M, Andersson DI, Sandegren L. Combinations of mutations in envZ, ftsl, mrdA, acrB and acrR can cause high-level carbapenem resistance in Escherichia coli. J Antimicrob Chemother. 2016;71(5):1188-98.

15. Webber MA, Talukder A, Piddock LJ. Contribution of mutation at amino acid 45 of AcrR to acrB expression and ciprofloxacin resistance in clinical and veterinary Escherichia coli isolates. American Society for Microbiology. 2005;49(10):4390-2.

16. Olliver A, Valle M, Chaslus-Dancla E, Cloeckaert A. Overexpression of the multidrug efflux operon acrEF by insertional activation with IS1 or IS10 elements in Salmonella enterica serovar typhimurium DT204 acrB mutants selected with fluoroquinolones. Antimicrob Agents Chemother. 2005;49(1):289-301. 
17. Kim YJ, Im SY, Lee JO, Kim OB. Potential swimming motility variation by AcrR in Escherichia coli. J Microbiol Biotechnol. 2016;26(10):1824-8.

18. Hadfield J, Croucher NJ, Goater RJ, Abudahab K, Aanensen DM, Harris SR. Phandango: an interactive viewer for bacterial population genomics. Bioinformatics. 2017. [Epub ahead of print]

19. Ma D, Alberti M, Lynch $C$, Nikaido H, Hearst JE. The local repressor AcrR plays a modulating role in the regulation of acrAB genes of Escherichia coli by global stress signals. Mol Microbiol. 1996;19(1):101-12.

20. Su CC, Rutherford DJ, Yu EW. Characterization of the multidrug efflux regulator AcrR from Escherichia coli. Biochem Biophys Res Commun. 2007:361(1):85-90

21. Li M, Gu R, Su CC, Routh MD, Harris KC, Jewell ES, et al. Crystal structure of the transcriptional regulator AcrR from Escherichia coli. J Mol Biol. 2008; 374(3):591-603.

22. Gray MJ, Wholey W. Jakob U. The Journal of Biological Chemistry: NemR is a bleach-sensing transcription factor; 2013.

23. Schumacher MA, Zeng W. Structures of the nucleoid occlusion protein SImA bound to DNA and the C-terminal domain of the cytoskeletal protein FtsZ. Proc Natl Acad Sci USA. 2016;113(18):4988-93.

24. Du S, Lutkenhaus J. SImA Antagonism of FtsZ Assembly Employs a Twopronged Mechanism like MinCD. PloS Genet. 2014;10(7). [Epub ahead of print]

25. Klancher CA, Hayes CA, Dalia AB. The nucleoid occlusion protein SImA is a direct transcriptional activator of chitobiose utilization in Vibrio cholerae. PloS Genet. 2017;13(7):e1006877.

26. Yamanaka Y, Shimada T, Yamamoto K, Ishihama A. Transcription factor $\mathrm{CecR}(\mathrm{YbiH})$ regulates a set of genes affecting the sensitivity of Escherichia coli against cefoperazone and chloramphenicol. Microbiology. 2016:162(7):1253-64.

27. Rkenes TP, Lamark T, Strøm AR. DNA-binding properties of the Betl repressor protein of Escherichia coli: the inducer choline stimulates BetlDNA complex formation. J Bacteriol. 1996;178(6):1663-70.

28. Masi M, Pages JM, Villard C, Pradel E. The eefABC multidrug efflux pump operon is repressed by H-NS in Enterobacter aerogenes. J Bacteriol. 2005;187(11):3894-7

29. Masi M, Pages JM, Pradel E. Production of the cryptic EefABC efflux pump in Enterobacter aerogenes chloramphenicol-resistant mutants. J Antimicrob Chemother. 2006;57(6):1223-6.

30. Zhang YM, Marrakchi H, Rock CO. The FabR (YijC) transcription factor regulates unsaturated fatty acid biosynthesis in Escherichia coli. J Biol Chem. 2002;277(18):15558-65.

31. Yamasaki S, Nakashima R, Sakurai K, Baucheron S, Giraud E, Doublet B, et al. Crystal structure of the multidrug resistance regulator RamR complexed with bile acids. Sci Rep. 2019;9(1):177.

32. Yamasaki S, Nikaido E, Nakashima R, Sakurai K, Fujiwara D, Fujii I, et al. The crystal structure of multidrug-resistance regulator RamR with multiple drugs. Nat Commun. 2013:4:2078.

33. Ricci V, Busby SJ, Piddock LJ. Regulation of RamA by RamR in Salmonella enterica serovar typhimurium: isolation of a RamR superrepressor. Antimicrob Agents Chemother. 2012;56(11):6037-40.

34. Shimada T, Hirao K, Kori A. RutR is the uracil/thymine-sensing master regulator of a set of genes for synthesis and degradation of pyrimdines. Mol Microbiol. 2007:66(3):744-57.

35. Nguyen Le Minh P, de Cima S, Bervoets I, Maes D, Rubio V, Charlier D. Ligand binding specificity of RutR, a member of the TetR family of transcription regulators in Escherichia coli. FEBS Open Bio. 2015;5:76-84.

36. Aleksandrov A, Schuldt L, Hinrichs W, Simonson T. Tetracycline-tet repressor binding specificity: insights from experiments and simulations. Biophys J. 2009:97(10):2829-38.

37. Blanco C, Ritzenthaler P, Mata-Gilsinger M. Negative dominant mutations of the uidR gene in Escherichia coli: genetic proof for a cooperative regulation of uidA expression. Genet. 1986;112(2):173-82.

38. Shimada T, Katayama Y, Kawakita S, Ogasawara H, Nakano M, Yamamoto $K$, et al. A novel regulator RcdA of the csgD gene encoding the master regulator of biofilm formation in Escherichia coli. Microbiologyopen. 2012;1(4):381-94.

39. Mermod M, Magnani D, Solioz M, Stoyanov JV. The copper-inducible ComR $(\mathrm{YcfQ})$ repressor regulates expression of ComC (YcfR), which affects copper permeability of the outer membrane of Escherichia coli. Biometals. 2012; 25(1):33-43.
40. Hwang J, Mattei LM, VanArendonk LG, Meneely PM, Okeke IN. A pathoadaptive deletion in an enteroaggregative Escherichia coli outbreak strain enhances virulence in a Caenorhabditis elegans model. Infect Immun. 2010;78(9):4068-76.

41. Ma Q, Zhang G, Wood TK. Escherichia coli BdcA controls biofilm dispersal in Pseudomonas aeruginosa and Rhizobium meliloti. BMC Res Notes. 2011;4:447.

42. Tonthat NK, Arold ST, Pickering BF, Van Dyke MW, Liang S, Lu Y, et al. Molecular mechanism by which the nucleoid occlusion factor, SImA, keeps cytokinesis in check. EMBO J. 2011;30(1):154-64.

43. Merhej V, Royer-Carenzi M, Pontarotti P, Raoult D. Massive comparative genomic analysis reveals convergent evolution of specialized bacteria. Biol Direct. 2009:4:13.

44. Miravet-Verde S, Llorens-Rico V, Serrano L. Alternative transcriptional regulation in genome-reduced bacteria. Curr Opin Microbiol. 2017:39:89-95.

45. Moradali MF, Ghods S, Rehm BH. Pseudomonas aeruginosa lifestyle: a paradigm for adaptation, survival, and persistence. Front Cell Infect Microbiol. 2017;7:39.

46. Glover B, Wentzel J, Jenkins A, Van Vuuren M. The first report of Escherichia fergusonii isolated from non-human primates, in Africa. One Health. 2017;3:70-5.

47. Yamamoto D, Hernandes RT, Liberatore AM, Abe CM, Souza RB, Romao FT, et al. Escherichia albertii, a novel human enteropathogen, colonizes rat enterocytes and translocates to extra-intestinal sites. PLoS One. 2017;12(2):e0171385.

48. Inglis TJ, Merritt AJ, Bzdyl N, Lansley S, Urosevic MN. First bacteraemic human infection with Escherichia albertii. New Microbes New Infect. 2015:8:171-3.

49. Savini V, Catavitello C, Talia M, Manna A, Pompetti F, Favaro M, et al. Multidrug-resistant Escherichia fergusonii: a case of acute cystitis. J Clin Microbiol. 2008;46(4):1551-2.

50. Brown NL, Stoyanov JV, Kidd SP, Hobman JL. The MerR family of transcriptional regulators. FEMS Microbiol Rev. 2003;27(2-3):145-63.

51. Shimada $T$, Ogasawara $H$, Ishihama A. Single-target regulators form a minor group of transcription factors in Escherichia coli K-12. Nucleic Acids Res. 2018:46(8):3921-36

52. Rogers JM, Bulyk ML. Diversification of transcription factor-DNA interactions and the evolution of gene regulatory networks. WIREs Syst Biol Med. 2018; 10(5). [Epub ahead of print]

53. Cooper TF, Rozen DE, Lenski RE. Parallel changes in gene expression after 20,000 generations of evolution in Escherichia coli. PNAS. 2003;100(3):1072-7.

54. Voordeckers K, Pougach K, Verstrepen KJ. How do regulatory networks evolve and expand throughout evolution? Curr Opin Biotechnol. 2015;34: 180-8.

55. Chiu SK, Huang LY, Chen H, Tsai YK, Liou CH, Lin JC, et al. Roles of ramR and tet(A) Mutations in Conferring Tigecycline Resistance in CarbapenemResistant Klebsiella pneumoniae Clinical Isolates. Antimicrob Agents Chemother. 2017;61(8).

56. Henssler EM, Bertram R, Wisshak S, Hillen W. Tet repressor mutants with altered effector binding and allostery. FEBS J. 2005;272(17):4487-96.

57. Mitchell AL, Attwood TK, Babbitt PC, Blum M, Bork P, Bridge A, et al. InterPro in 2019: improving coverage, classification and access to protein sequence annotations. Nucleic Acids Res. 2019;47(D1):D351-D60.

58. Altschul SF, Gish W, Miller W, Myers EW, Lipman DJ. Basic local alignment search tool. J Mol Biol. 1990;215(3):403-10.

59. Goujon M, McWilliam H, Li W, Valentin F, Squizzato S, Paern J, et al. A new bioinformatics analysis tools framework at EMBL-EBI. Nucleic Acids Res. 2010;38(2). [Epub ahead of print]

60. Larkin MA, Blackshields G, Brown NP, Chenna R, McGettigan PA, McWilliam $H$, et al. Clustal W and Clustal X version 2.0. Bioinformatics. 2007;23(21):2947-8

61. Edgar RC. MUSCLE: multiple sequence alignment with high accuracy and high throughput. Nucleic Acids Res. 2004;32(5):1792-7.

62. Kumar S, Stecher G, Tamura K. MEGA7: molecular evolutionary genetics analysis version 7.0 for bigger datasets. Mol Biol Evol. 2016;33(7):1870-4

\section{Publisher's Note}

Springer Nature remains neutral with regard to jurisdictional claims in published maps and institutional affiliations. 